\title{
Research on the characteristics of Klein-Cook parameter and diffraction efficiency of acousto-optic interaction for low-frequency ultrasonic in the liquid
}

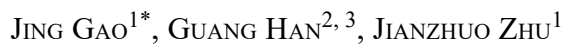 \\ ${ }^{1}$ Hebei Key Laboratory of Microstructure Materials Physics, College of Science, \\ Yanshan University, Qinhuangdao, Hebei 066004, China \\ ${ }^{2}$ Mechanical and Electrical Engineering Department, \\ Hebei Construction Material Vocational and Technical College, \\ Qinhuangdao, Hebei 066004, China \\ ${ }^{3}$ College of Mechanical Engineering, Yanshan University, \\ Qinhuangdao, Hebei 066004, China \\ ${ }^{*}$ Corresponding author: gaojing986@126.com
}

\begin{abstract}
We have investigated the characteristics of acousto-optic interaction for low-frequency ultrasonic wave in a liquid. Based on the coupling wave equation of acousto-optic interaction, the diffraction light characteristics for normal incidence at small parameter $Q$ have been discussed. The parameter $Q$ with respect to acousto-optic interaction length, ultrasonic frequency, water temperature, and the concentration of sucrose solution have been analyzed, which is an important physical quantity and reflects the degree of mismatch in the acousto-optic interaction. The diffraction efficiencies for different parameters $Q$, incident angles and phase shifts have been calculated. The results of our work provide theoretical basis for further study of the acousto-optic effect in the liquid.
\end{abstract}

Keywords: acousto-optic interaction, Klein-Cook parameter, Raman-Nath diffraction, diffraction efficiency.

\section{Introduction}

When ultrasonic wave propagates in the medium as a longitudinal wave, its sound pressure makes the density of the medium obvious periodical change. Thus, the refractive index of the medium is also changed periodically. Diffraction occurs when incident light passes through this modulated medium [1, $\underline{2}$ ]. In 1967, KLEIN and CoOK [ $\underline{3}]$ introduced the parameter $Q$ to distinguish two diffraction types: Raman-Nath and Bragg diffractions. With the development of laser technology, the theory and the application 
of the acousto-optic effect have been promoted rapidly. By using the principle of acousto -optic interaction, various acousto-optic devices have been made, such as acousto-optic modulator, acousto-optic $Q$ switch, tunable filter, acousto-optic deflector [ $\underline{4}-\underline{8}]$. These devices can control the direction, intensity and frequency of laser beam quickly and effectively, and convert electrical signals into optical signals in real time.

WINDELS and LEROY [9] presented a new general analytical formula which described the interaction of a Gaussian laser beam of arbitrary width with a sound column, valid under Raman-Nath conditions. BogdAN et al. [10] put forward an acousto-optical analog microwave spectrometer (AOS) with a serial-type acousto-optical unevenness (AO) interaction of less than $3 \mathrm{~dB}$ in the frequency range 500 to $1750 \mathrm{MHz}$ central frequency. FERRIA et al. [11] presented and discussed an acousto-optic technique for measuring the index of refraction of transparent liquid materials. The refractive index of liquid was determined by the spatial frequency measurement of the diffractive pattern. In their work, the refractive indexes of acetone, ethanol and distilled water were measured, and the obtained results were in a good agreement with results of other researchers in the field. Two years later, they found that acoustic impedances of the binary solutions have an influence on the diffraction efficiency. The dependences of velocities and diffraction efficiencies on the concentrations of liquids (ethanol and acetone) were nonlinear and symmetrical with respect to each other [12]. XUE et al. [13] presented a new method to measure the velocity of sound in pure water and seawater using Raman-Nath diffraction caused by the acousto-optic effect between the optical frequency comb and the ultrasonic pulse, and compared with the reference value, the measurement uncertainty can be well below $0.03 \mathrm{~m} / \mathrm{s}$. GiULIANO et al. [14] achieved an underwater wireless acousto-optic waveguide (UWAOW) using the acousto-optic effect, which was based on a localised change in refractive index of seawater induced by a high sound pressure level. In their work, the speeds of sound underwater with different temperatures and depths, and the refractive index of water as a function of the externally applied acoustic pressure were studied.

Although there are many researches on acousto-optic effect, the characteristics of acousto-optic interaction in the liquid have barely been investigated. In this regard, our work is devoted to the study of the changes of Klein-Cook parameter and diffraction efficiencies under different acousto-optic conditions, and then the characteristics of diffraction light at different orders are found.

\section{Principle of acousto-optic effect}

The periodic distribution of refractive index in the medium caused by ultrasonic wave can be equivalent to phase grating. The intensity, frequency and direction of diffraction light after the beam passes through this medium vary with the refractive index distribution in the medium. There are two types: normal acousto-optic effect and abnormal acousto-optic effect for acousto-optic interaction. In isotropic medium, the diffraction light has the same polarization state as the incident light, thus the acousto-optic interaction is called normal acousto-optic effect. By contrast, the polarization state of the 
Electroacoustic transducer

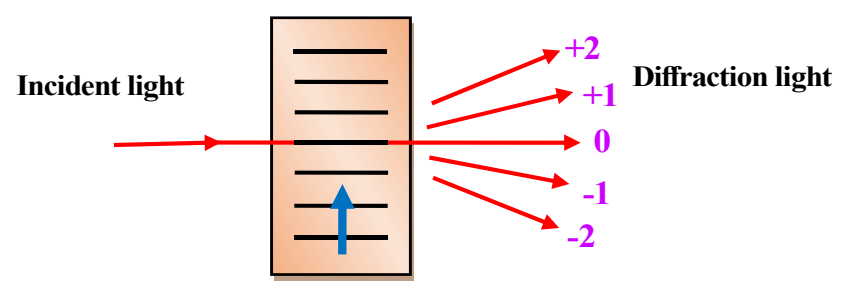

Ultrasonic wave

Electroacoustic transducer

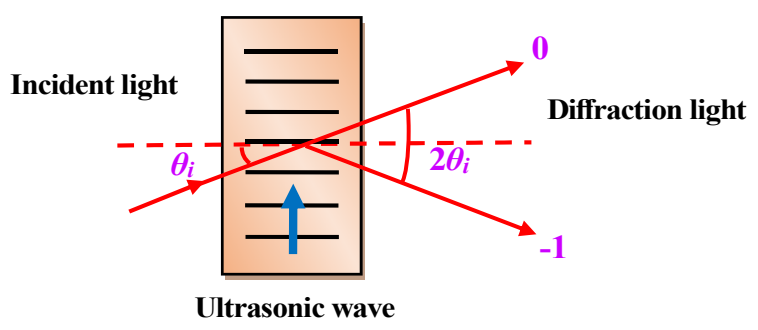

a

Fig. 1. Acousto-optic effect: (a) Raman-Nath diffraction, and (b) Bragg diffraction.

diffraction light differs from that of the incident light in anisotropic medium, thus the acousto-optic interaction in this case is called abnormal acousto-optic effect. The normal acousto-optic effect is studied in this paper. Raman-Nath diffraction is generated for low-frequency ultrasonic wave, short length of acousto-optic interaction region and incident light normal to the propagation direction of the ultrasonic field (Fig. 1a). Under this condition, multi-order diffraction light with the characteristics of diffraction fringes of each order distributed on both sides of the zeroth-order fringe symmetrically, and diffraction intensities of each order decreasing gradually. On the contrary, Bragg diffraction is generated for high frequency of ultrasonic wave, long length of acousto-optic interaction region and incident light at a certain angle to the acoustic wave front. The diffraction light is not symmetrical, only zeroth order, positive or negative first order depending on the direction of the incident light for Bragg regime (Fig. 1b). The interaction of incident light with low-frequency ultrasonic wave is a focused study in our work.

Sound wave is an elastic wave. There is an elastic deformation, in the medium of which the optical property changes, that is, the refractive index varies as the acoustic wave propagates through the medium. According to the principle of acousto-optic effect, the distribution of refractive index in the medium caused by ultrasonic wave across $x$ direction can be represented by

$$
n(x, t)=n_{0}+\Delta n \sin \left(\omega_{\mathrm{s}} t-k_{\mathrm{s}} x\right)
$$

In this expression, $n_{0}$ is the refractive index of the medium in the absence of ultrasonic wave, $\Delta n$ is the variation of refractive index owing to the acoustic strain, and $\omega_{\mathrm{s}}, k_{\mathrm{s}}$ are 
the circular frequency and wave number of the ultrasonic wave, respectively. The parameter $\Delta n$ is related to the acoustical pressure $p$ by [ $\underline{3}]$

$$
\Delta n=\left(\frac{\partial n}{\partial p}\right)_{\mathrm{s}} p
$$

where $(\partial n / \partial p)_{\mathrm{s}}$ is the adiabatic piezooptic coefficient.

Considering that the refractive index variation of the medium is very small, and light travels much faster than sound in the medium, the time factor can be omitted. Thus, the distribution of refractive index in the medium along $x$ axis produced by an ultrasonic wave can be reduced to

$$
n(x)=n_{0}-\Delta n \sin \left(k_{\mathrm{s}} x\right)
$$

The sound wave propagates across $x$ axis with width $L$, wavelength $\lambda_{\mathrm{s}}$, wave number $k_{\mathrm{s}}$ in the acoustic-optical medium, and the incident light with wave number $k_{\mathrm{i}}$ in the air pointing to $y$ axis, as indicated in Fig. 2 .

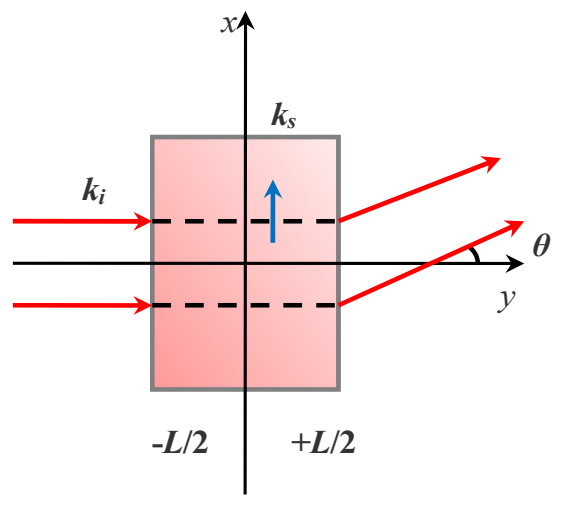

Fig. 2. Incident light normal to the sound field.

For a sinusoidal sound field, if the light irradiates the acousto-optic medium at an incident angle $\theta$, the coupling wave equation can be written as $[\underline{3}, \underline{15}]$

$$
\frac{\mathrm{d} E_{m}(x)}{\mathrm{d} z}+\frac{\delta}{2 L}\left[E_{m-1}(x)-E_{m+1}(x)\right]=i \frac{m Q}{2 L}(m-2 \alpha) E_{m}(x)
$$

where $m$ represents the order of diffraction light, $\delta$ denotes the additional phase shift due to the change in the refractive index of the medium, $Q$ is Klein-Cook parameter, $\alpha$ denotes a physical quantity related to the incident angle.

The additional phase shift $\delta$ is in the form

$$
\delta=k_{\mathrm{i}}\left(\frac{\partial n}{\partial p}\right)_{\mathrm{s}} p L
$$


From Eqs. (2) and (5), $\delta$ can be written as

$$
\delta=\frac{2 \pi}{\lambda_{\mathrm{i}} \cos \theta} \Delta n L \approx \frac{2 \pi}{\lambda_{\mathrm{i}}} \Delta n L
$$

where $\lambda_{\mathrm{i}}$ denotes the wavelength of incident light in the air.

Klein-Cook parameter $Q$ and $\alpha$ depending on the incident angle are represented by

$$
\begin{aligned}
& Q=\frac{k_{\mathrm{s}}^{2} L}{n_{0} k_{\mathrm{i}} \cos \theta} \approx \frac{2 \pi \lambda_{\mathrm{i}} L}{n_{0} \lambda_{\mathrm{s}}^{2}}=\frac{2 \pi \lambda L}{\lambda_{\mathrm{s}}^{2}} \\
& \alpha=\frac{n_{0} k_{\mathrm{i}}}{k_{\mathrm{s}}} \sin \theta=\frac{k}{k_{\mathrm{s}}} \sin \theta
\end{aligned}
$$

where $\lambda, k$ are the wavelength and wave number of incident light in the medium. The parameter $Q$ depending on $\lambda_{\mathrm{i}}, \lambda_{\mathrm{s}}, L, n_{0}$ is a normally constant in a given experimental situation. The general nature of the diffraction process is described by this parameter.

The free wave vector of the diffraction light excited by the polarized wave is represented by $\mathbf{k}_{m}$. In the case of momentum mismatch $\left(\Delta k_{m} \neq 0\right)$, when the light is incident at arbitrary angle, the $m$-th diffraction light excited by the polarized wave does not meet the same phase condition, thus the $m$-th diffraction light disappears. The magnitude of the vector $\Delta k_{m}$ is

$$
\Delta k_{m}=\frac{m Q}{2 L}(m-2 \alpha)
$$

From Eq. (9), $\Delta k_{m} \propto Q$, therefore the parameter $Q$ reflects the degree of mismatch in the acousto-optic interaction. The phase shift $\delta$ reflects the strength of the acousto -optic interaction.

Under the condition $Q \ll 1$, Eq. (4) can be reduced to the form

$$
\frac{\mathrm{d} E_{m}(x)}{\mathrm{d} z}+\frac{\delta}{2 L}\left[E_{m-1}(x)-E_{m+1}(x)\right]=-i \frac{m Q}{L} \alpha E_{m}(x)
$$

Using the recursion relation for the $m$-th-order Bessel function of the first kind

$$
J_{m}^{\prime}(z)=\frac{1}{2}\left[J_{m-1}(z)-J_{m+1}(z)\right]
$$

the solution of the equation that satisfies the boundary conditions $\left(E_{0}(0)=E_{0}\right.$, $\left.E_{m}(0)=0\right)$ is

$$
E_{m}(x)=E_{0} \exp \left(-i \frac{m Q \alpha}{2 L} x\right) J_{m}\left[\frac{2 \delta}{Q \alpha} \sin \left(\frac{Q \alpha}{2 L} x\right)\right]
$$


At $x=L$, the $m$-th diffraction efficiency is

$$
\eta_{m}=\left|\frac{E_{m}(L)}{E_{0}}\right|^{2}=J_{m}^{2}\left[\delta \frac{\sin (Q \alpha / 2)}{Q \alpha / 2}\right]
$$

At normal incidence $\alpha=0$, Eq. (13) becomes

$$
\eta_{m}=J_{m}^{2}(\delta)
$$

Hence, the intensity of the $m$-th diffraction light can be described by

$$
I_{m} \propto J_{m}^{2}(\delta)
$$

The diffraction mentioned above is called Raman-Nath diffraction, and the medium disturbed by the ultrasonic wave is similar to a plane phase grating.

Equation (4) no longer has simple analytic solutions in the case of $Q$ not much larger than 1 and not much less than 1. A matrix series method is used to solve Eq. (4) for non-Raman-Nath type, and the diffraction efficiency is calculated on this basis. The physical model is completely transformed to pure number solutions, which cannot only reduce the parameters, but also avoid some problems caused by solving Eq. (4) directly. In the actual acousto-optic interaction, the diffraction order is finite, so the higher order can be ignored. Therefore the infinite equation can be truncated to an ordinary differential equation of finite first order.

When the incident light is normal to the direction of ultrasonic propagation, the diffraction angle for the maximum intensity of diffraction light is determined by

$$
\sin \theta_{m}= \pm m \frac{k_{\mathrm{s}}}{k_{\mathrm{i}}}= \pm m \frac{\lambda_{\mathrm{i}}}{\lambda_{\mathrm{s}}}
$$

Equation (16) can also be regarded as the grating equation of phase grating, and the grating constant is the ultrasonic wavelength in the acousto-optic medium.

When the diffraction angle caused by acousto-optic interaction satisfies Eq. (16) but $J_{m}^{2}(\delta)=0$, the diffraction fringe of maximum position disappears. Figure 3 shows this phenomenon that the diffraction light at the first and second order disappears when $\delta$ are 3.84 and 5.14, respectively. The diffraction light at zeroth order disappears when $\delta$ are 2.405 and 5.520. That is to say, although the first-order diffraction efficiency is low, the light energy can all be transferred to the higher-order diffraction light when $\delta$ is set to an appropriate value. If $\delta$ is 1.85 , the diffraction intensity for the first order achieves the maximum with the diffraction efficiency of $33.85 \%$. If $\delta$ is 3.06 , the diffraction intensity for the second order achieves the maximum with the diffraction efficiency of $23.67 \%$. Figure 3 also shows the diffraction efficiency of diffraction order versus the phase shift in the ideal Raman-Nath diffraction with $Q=0$. 


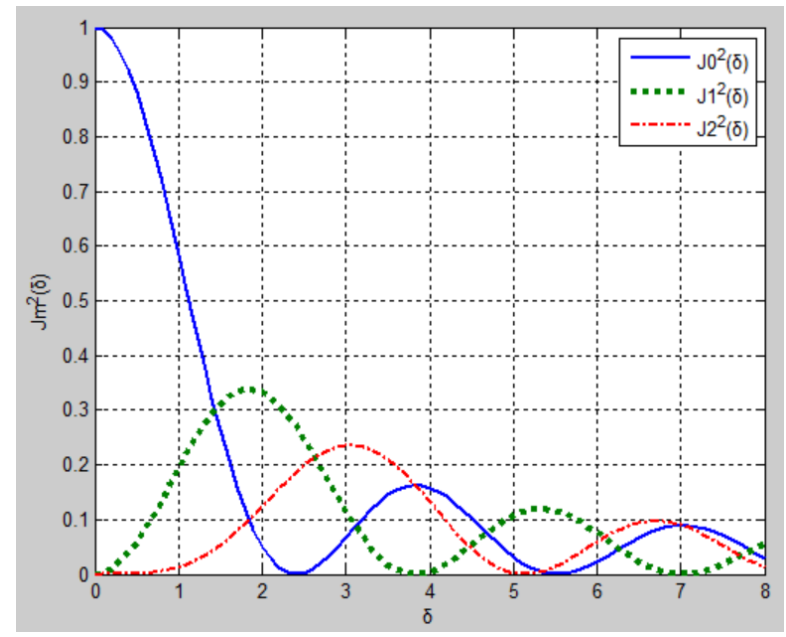

Fig. 3. Graph of a square of the Bessel function at different diffraction orders.

According to Eq. (6), the phase shift $\delta$ is determined by the wavelength of incident light $\lambda_{\mathrm{i}}$, the variation of refractive index $\Delta n$, and the acousto-optic interaction region length $L$. Therefore, by adjusting the parameters of $\lambda_{\mathrm{i}}, \Delta n$ and $L$, the diffraction intensity is made maximum for a certain order or disappeared for zeroth order.

\section{Characteristics of acousto-optic interaction in the liquid}

\subsection{Analysis of parameter $Q$}

Supposing that the ultrasonic wave is propagating through water, and fixing the incident light wavelength at $632.8 \mathrm{~nm}$ (He-Ne laser), the curves of Klein-Cook parameter $Q$ for different interaction lengths, ultrasonic frequencies, water temperatures were plotted (Fig. 4). The propagation velocities of ultrasonic wave in water at different temperatures were measured by the experimental system for sound velocity consisting of an ultrasonic experimental apparatus, signal source, and dual trace oscilloscope. The experimental device was put into the tank, and the water in the tank was 1-2 cm higher than the top of the transducer. The phase comparison method was used in the experiment. To control the water temperature, a digital display intelligent thermostat was used whose temperature range is from $-50^{\circ} \mathrm{C}$ to $110^{\circ} \mathrm{C}$. On the basis of the above work, the parameters $Q$ under different acousto-optic conditions were obtained. Figures $4 \mathbf{a}$ and $4 \mathbf{b}$, the temperature of water at $20^{\circ} \mathrm{C}$, show the influence of the acousto-optic interaction region length and the ultrasonic frequency on parameter $Q$. As the interaction region length increases in the range of 10 to 50 millimeters, the ultrasonic wave with higher frequency generates greater influence on parameter $Q$ of which the change range is larger. If the frequency changes in the range of 1 to $3 \mathrm{MHz}$, the parameters $Q$ corre- 
sponding to different interaction lengths change little. When the ultrasonic frequency is higher than $4 \mathrm{MHz}$, the parameter $Q$ related to large length grows faster than that of small length. The maximum value of parameter $Q$ is close to 7 , thus the diffraction regime may change as the interaction length and frequency change.

In Figs. $4 \mathbf{c}$ and $4 \mathbf{d}$, the parameter $Q$ at the water temperature of $10^{\circ} \mathrm{C}$ is 1.135 times that of temperature at $60^{\circ} \mathrm{C}$. As the water temperature raises, $Q$ value decreases gradually, but its change is small. Under the condition of ultrasonic frequency at $2 \mathrm{MHz}$, $Q$ is very small although the interaction length increases by nearly three times. Thus, Raman-Nath diffraction occurs for low-frequency ultrasonic wave incident into the water. Under the condition that the interaction length is $15 \mathrm{~mm}$, the parameter $Q$ is less than 0.3 when the ultrasonic frequency is no more than $4 \mathrm{MHz}$, and this diffraction is Raman-Nath diffraction regime. For the frequency of $10 \mathrm{MHz}$, parameter $Q$ is nearly 2 in the range of water temperature from $10^{\circ} \mathrm{C}$ to $60^{\circ} \mathrm{C}$.

The refractive index and velocity of ultrasonic wave in the liquid vary with the concentration of solution. Taking sucrose solution as an example, the refractive indexes of different concentrations were measured using Abbe refractometer that can quickly

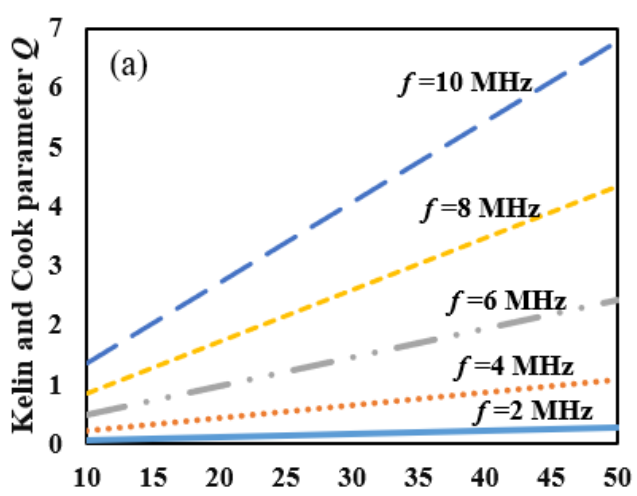

Acusto-optic interaction length $L / \mathrm{mm}$

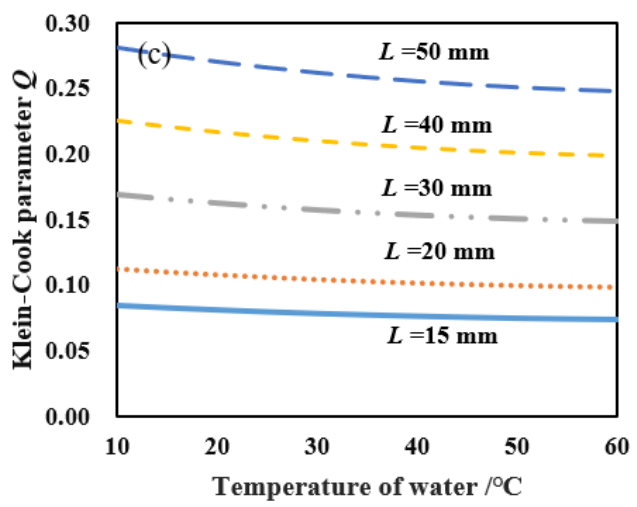

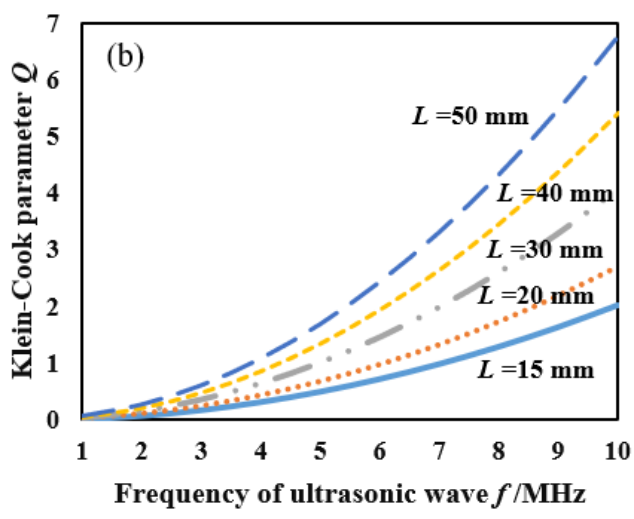

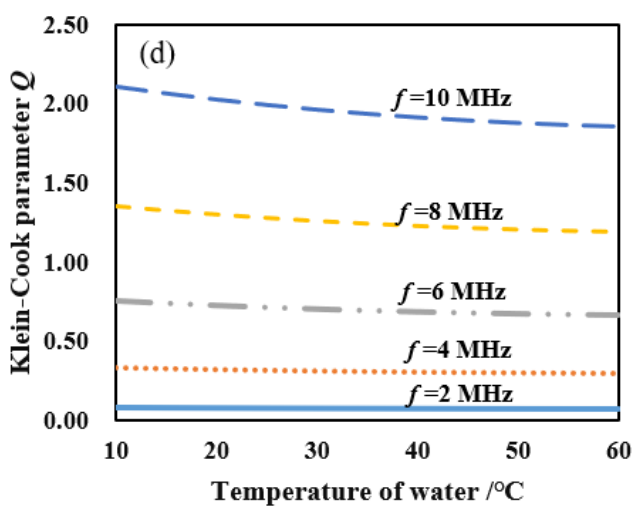

Fig. 4. Variation curves of parameter $Q$ for $\lambda=623.8 \mathrm{~nm}$ in water: $(\mathbf{a}, \mathbf{b}) t=20^{\circ} \mathrm{C}$, (c) $f=2 \mathrm{MHz}$, and (d) $L=15 \mathrm{~mm}$. 


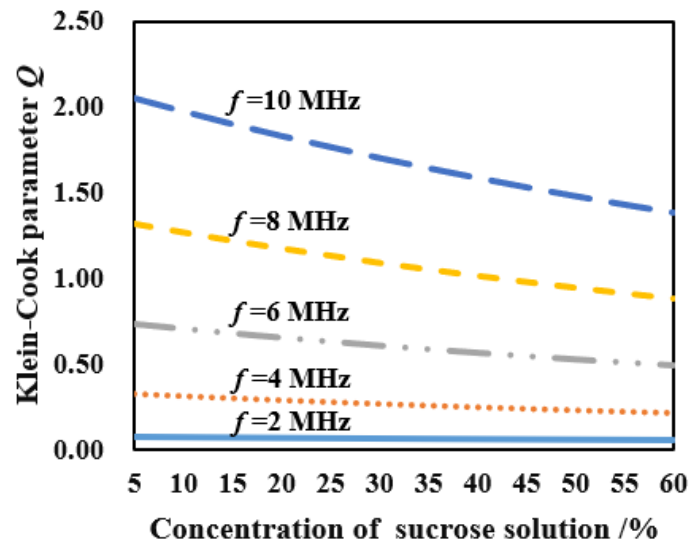

Fig. 5. Changes of parameter $Q$ at different concentrations of sucrose solution.

and accurately measure the refractive index of transparent, translucent liquid and solid materials. The velocities of ultrasonic wave in the sucrose solution with different concentrations were measured by a sound velocimeter using the phase comparison method. The curves of $Q$ with respect to the concentration were plotted (Fig. 5). Moreover, the temperature of liquid was $20^{\circ} \mathrm{C}$ and the interaction length was $15 \mathrm{~mm}$. As the concentration of sucrose solution increases, $Q$ parameter decreases. The change of $Q$ is so small that it can be ignored at $f=2 \mathrm{MHz}$. However, the higher the ultrasonic frequency, the more obvious the change. In other words, $Q$ is sensitive to the change in concentration for high frequency sound wave, thus the diffraction regime is also affected.

\subsection{Calculation of diffraction efficiency}

In this section, using the results derived from numerical solutions, the diffraction efficiencies for different parameters $Q$, phase shifts and incident angles are studied. The refractive index in liquid is changed due to the ultrasonic drive. By matching the ultrasonic feed parameters and acoustic power, $\Delta n$ can be adjusted, and then $\delta$ is also changed. Based on the previous section, $Q$ parameter is low when the ultrasonic frequency is $2 \mathrm{MHz}$. At the moment, the acousto-optic interaction is typical Raman-Nath diffraction. The diffraction efficiencies were analyzed at this frequency with the water temperature of $20^{\circ} \mathrm{C}$ and $Q$ parameter of 0.1 (Fig. 6). From Fig. 6, the diffraction light consists of 0 th order and \pm 1 st order stripes, and the light intensity is concentrated at 0 th order when the incident angle is $2^{\circ}$. The diffraction efficiency of 0 th order increases rapidly while the diffraction efficiencies of \pm 1 st order and \pm 2 nd order decrease sharply when the incident angle increases to $1.8^{\circ}$. No matter what phase shift is, the sum of maximum diffraction efficiencies at \pm 1 st and \pm 2 nd order is about $90 \%$. The distribution of incident angle with zeroth-order diffraction efficiency greater than $30 \%$ is: when $\delta=2$, the range of incident angle is from $-1^{\circ}$ to $1^{\circ}$; when $\delta=3$, the range of incident angle is from $1^{\circ}$ to $1.4^{\circ}$ and from $-1.4^{\circ}$ to $-1^{\circ}$; when $\delta=4$, the range of incident angle is from $1.4^{\circ}$ to $1.6^{\circ}$ and from $-1.6^{\circ}$ to $-1.4^{\circ}$; when $\delta=5$, the range of incident angle is 

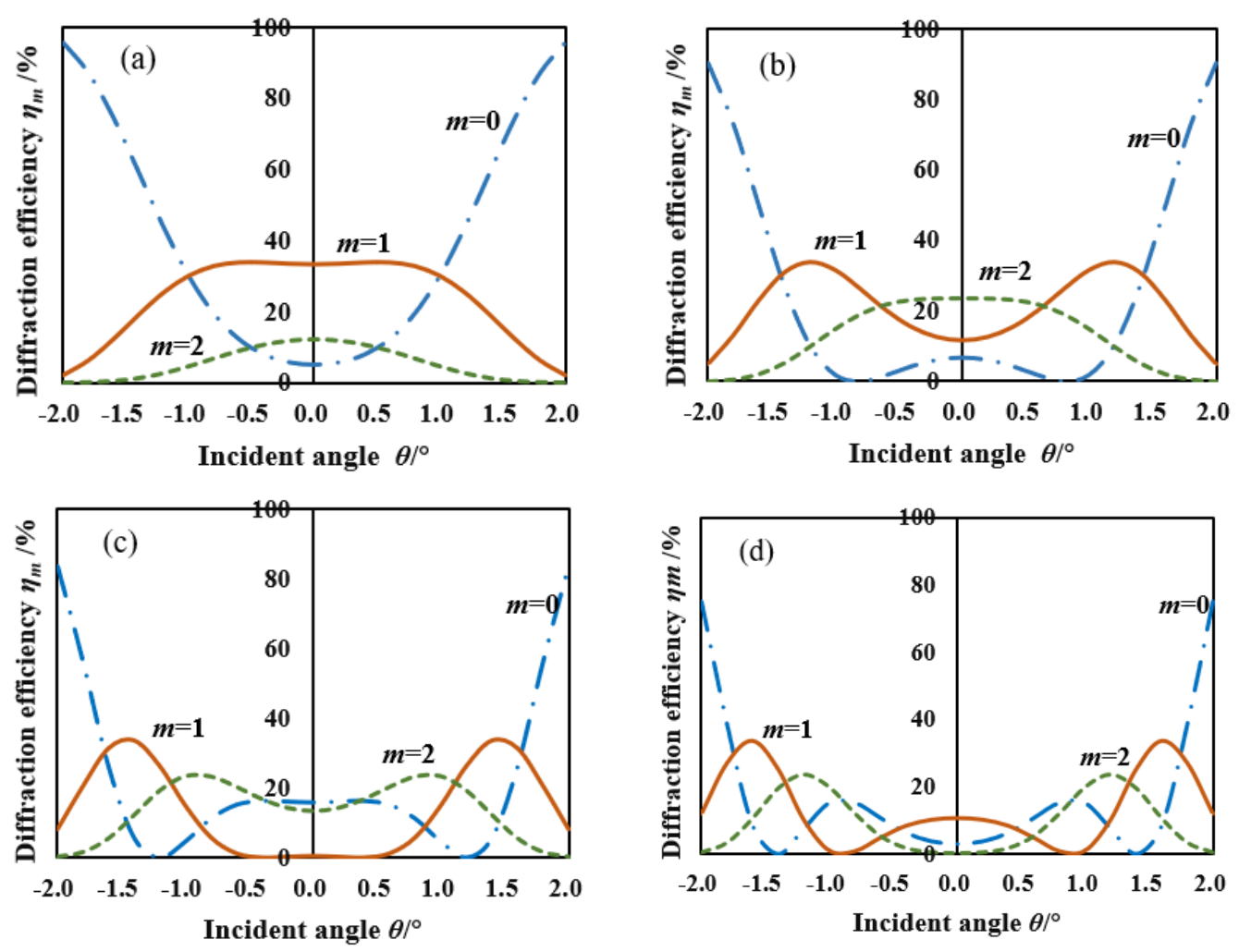

Fig. 6. Diffraction efficiencies at different incident angles with $Q=0.1$ : (a) $\delta=2$, (b) $\delta=3$, (c) $\delta=4$, and (d) $\delta=5$.

from $1.5^{\circ}$ to $1.6^{\circ}$ and $-1.6^{\circ}$ to $-1.5^{\circ}$. It can be seen that when the phase shift is small, the incident angle range is large for the first-order diffraction efficiency greater than $30 \%$. As the phase shift gets larger, the incident angle also gets larger corresponding to the maximum first-order diffraction efficiency.

$\mathrm{He}-\mathrm{Ne}$ laser irradiates vertically on the water disturbed by the ultrasonic wave. When $Q>1$, the diffraction efficiencies at zeroth order and the first order for different phase shifts were illustrated in Fig. 7.

Figure 7a shows the diffraction when $Q=2$. For the zeroth-order diffraction light, the diffraction efficiency is minimum but not zero under the condition that $\delta$ is close to 2 , and the diffraction efficiency is greater than $30 \%$ at $-4<\delta<4$. For the first-order diffraction light, the diffraction efficiency is maximum (about 36\%) at $\delta=2$, while is minimal but not zero at $\delta=4$. Compared with Fig. 3, it is found that the diffraction efficiency curves including 0th order and 1st order shift upward. In Fig. 7b, $Q=7$, the diffraction efficiency curves of 0 th order and 1 st order are all symmetric with respect to $\delta=2$ under the condition that $\delta$ is less than 4 . In addition, the zeroth-order diffraction efficiency is greater than $70 \%$, and the curve is concave, while the first-order diffraction efficiency is less than $15 \%$, and the curve is convex. Compared with Fig. 3, the 

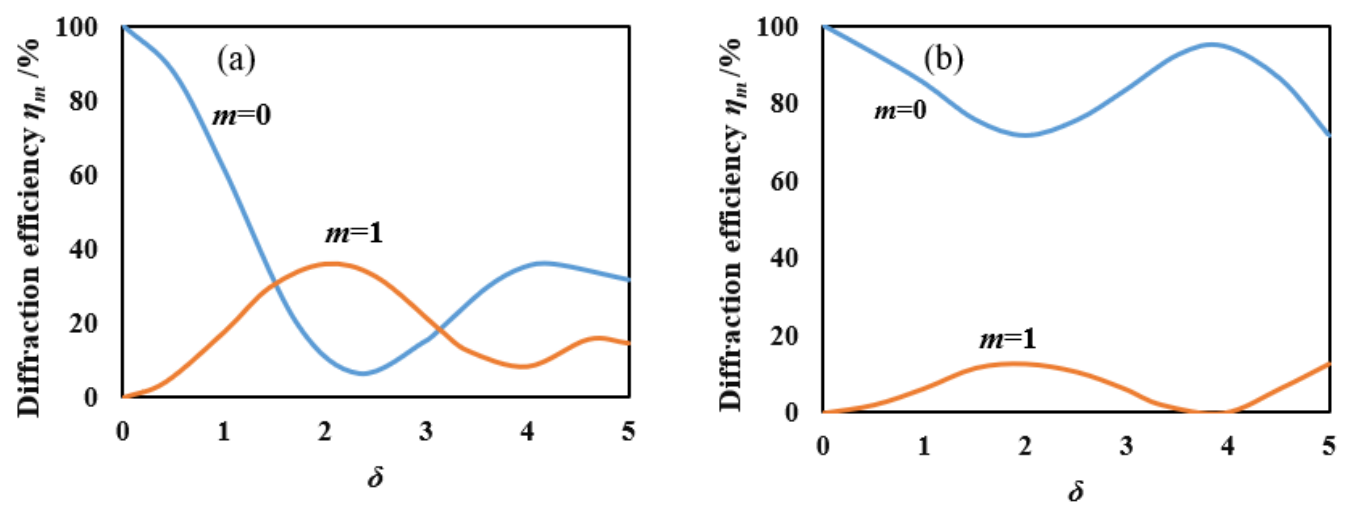

Fig. 7. Diffraction efficiencies at different phase shifts when incident light normal to water: (a) $Q=2$, and (b) $Q=7$.

diffraction intensity is stronger for 0 th order while weaker for 1st order. In this case, the diffraction is no longer Raman-Nath regime.

\section{Conclusions}

In this paper, we have investigated the characteristics of acousto-optic interaction in the liquid. For Raman-Nath regime, although the first-order diffraction efficiency is low, the light energy can all be transferred to the higher-order diffraction light when the phase shift is set to an appropriate value. Since Klein-Cook parameter $Q$ describes the general nature of diffraction process, we first analyze $Q$ in detail. The relations that parameter $Q$ varies with interaction length, ultrasonic frequency, water temperature and the concentration of sucrose solution are obtained. On the basis of the previously studied variation range of $Q$, the diffraction efficiencies for different parameters $Q$, phase shifts and incident angles have been calculated. The sum of maximum diffraction efficiencies at \pm 1 st and \pm 2 nd order is approximately $90 \%$ for $Q=0.1$. The adjustable range of incident angle is from $-1^{\circ}$ to $1^{\circ}$ for the phase shift of 2 to achieve the first -order diffraction efficiency more than $30 \%$. In the case of normal incident light, the maximum diffraction efficiency at 1 st order is about $36 \%$ for $Q=2$, which is improved compared with ideal Raman-Nath diffraction. The results of this paper provide theoretical basis for the further study of acousto-optic effect in the liquid.

Acknowledgment - The authors would like to acknowledge the financial support from Teaching Research and Reform Project of Yanshan University (Grant No. 2019XJJG19), and Natural Science Foundation of Hebei Province (Grant No. F2018203263).

\section{References}

[1] Mys O., Kostyrko M., Krupych O., Vlokh R., Anisotropy of the acousto-optic figure of merit for $\mathrm{LiNbO}_{3}$ crystals: isotropic diffraction, Applied Optics 54(27), 2015, pp. 8176-8186, DOI: 10.1364 / AO.54.008176. 
[2] Weng C., He Y., Acousto-optic interaction in Raman-Nath acousto-optic diffraction, European Physical Journal D 73(1), 2019, p. 1, DOI: 10.1140/epjd/e2018-90122-y.

[3] KLeIN W.R., CоOK B.D., Unified approach to ultrasonic light diffraction, IEEE Transactions on Sonics and Ultrasonics 14(3), 1967, pp. 123-134, DOI: 10.1109/T-SU.1967.29423.

[4] Yushrov K.B., Molchanov V.Y., Hyperspectral imaging acousto-optic system with spatial filtering for optical phase visualization, Journal of Biomedical Optics 22(6), 2017, article 066017, DOI: 10.1117/1.JBO.22.6.066017.

[5] Polschikova O.V., Machikhin A.S., Ramazanova A.G., Bratchenko I.A., Pozhar V.E., Danilycheva I.V., Katunina O.R., Danilychev M.V., An acousto-optic hyperspectral unit for histological study of microscopic objects, Optics and Spectroscopy 125(6), 2018, pp. 1074-1080, DOI: $10.1134 / \mathrm{S} 0030400 \mathrm{X} 19020188$.

[6] Antonov S.N., Acousto-optic deflector with heat removal from the piezotransducer by sound insulation of a heat radiator, Acoustical Physics 65(5), 2019, pp. 487-494, DOI: 10.1134/S1063771019050038.

[7] Fan Z., Wen Y., Shi X., Wu C.T., Chen X.Y., Jiang Y., Dai T.Y., LD double-end pumped dual-rod acousto-optic Q-switched Tm:LuAG laser, Infrared Physics and Technology 102, 2019, article 103022, DOI: 10.1016/j.infrared.2019.103022.

[8] Nikitin P.A., Gerasimov V.V., Voloshinov V.B., Acousto-optic modulation of terahertz radiation in liquefied sulfur hexafluoride at room temperature, Journal of Infrared, Millimeter, and Terahertz Waves 41, 2020, pp. 299-306, DOI: 10.1007/s10762-019-00660-7.

[9] Windels F.W., LEROY O., The acousto-optical interaction of narrow laser beams under Raman-Nath conditions, Journal of Optics A: Pure and Applied Optics 3(4), 2001, pp. S12-S17, DOI: 10.1088/ $1464-4258 / 3 / 4 / 353$.

[10] Bogdan O.V., Pavlenko V.K., Pashkevich G.A., Shkadarevich A.P., Acousto-optical analyzers of microwave broadband spectrum, 2013 23rd International Crimean Conference "Microwave \& Telecommunication Technology", September 8-14, 2013, Sevastopol, Ukraine, pp. 940-941.

[11] Ferria K., Laouar N., Bouaouadja N., Acousto-optic method for liquids refractometry, Optica Applicata 41(1), 2011, pp. 109-119.

[12] Ferria K., Griani L., LaOuar N., Acousto-optic method for quality control of water mixed with miscible liquids, Optics and Laser Technology 49, 2013, pp. 51-55, DOI: 10.1016/j.optlastec.2012.11.034.

[13] Xue B., Wang Z., Zhang K., Zhang H, Chen Y., Jia L., Wu H., Zhai J., Direct measurement of the sound velocity in seawater based on the pulsed acousto-optic effect between the frequency comb and the ultrasonic pulse, Optics Express 26(17), 2018, pp. 21849-21860, DOI: 10.1364/OE.26.021849.

[14] Giuliano G., Kent L.W.J., Laycock L.C., Underwater wireless acousto-optic waveguide (UWAOW), Proceedings of SPIE 10437, 2017, article 1043708, DOI: $10.1117 / 12.2286318$.

[15] KwIEк P., Diffraction of collinear correlated photon pairs by an ultrasonic wave within Raman-Nath and intermediate region, Ultrasonics 57, 2015, pp. 153-158, DOI: 10.1016/j.ultras.2014.11.005.

Received June 18, 2020 in revised form July 21, 2020 